\title{
A New Design of a Functional H Filter for Linear Singular Systems With an Additional Unknown Input and Bounded Disturbances
}

\author{
Hamzaoui Fatma, University of Monastir, Tunisia \& National Engineering School of Monastir, Tunisia \\ Khadhraoui Malek, National Engineering School of Monastir, Tunisia \\ Messaoud Hassani, National Engineering School of Monastir, Tunisia
}

\begin{abstract}
This paper proposes a design of a $H_{-} ¥$ filter for a functional of the system state that the authors call functional $\mathrm{H} ¥ ¥$ filter in time domain for linear singular systems with additional unknown inputs and bounded disturbances. So, this research proposes the estimation of a part of the unknown inputs which will be injected on the system state equation besides the functional state estimation. The design procedure is based on the unbiasedness of the estimation error using Lyapunov-Krasovskii stability theory and H_¥ criterion. The designed filter is characterized by a gain which is the solution of linear matrix inequalities (LMI). Numerical example is given to illustrate the proposed approach.
\end{abstract}

\section{KEYWORDS}

Bounded Disturbances, Functional Filter, H_¥ Criterion, Singular Systems, Unknown Input

\section{INTRODUCTION}

The reconstruction of the state vector is an inevitable step for control and diagnosis of many processes (Darouach, 2005; Khadhraoui, 2014). This topic has been investigated by many researchers during the last decades (Johnson, 1975; Hostetter, 1973) aiming the synthesis of a control scheme based on an observer gain. Then, the control law is expresses mainly in function of the output measurements. For this purpose, we propose the synthesis of an unknown input functional filter for linear singular systems affected by bounded disturbances and unknown inputs. This is motivated by the importance of such system which brings together three criteria (singular, unknown input and bounded disturbance) often present in most of physical process.

Also, the singularity has, recently, become the focus of several research activities (Dai, 1989; Darouach, 1995; Darouach, 2005) due to its importance in the modeling of physical systems. In fact singular system can be viewed as a generalization of standard linear systems as they contain a dynamical and algebraic part in the systems descriptions. Then the importance of such systems comes from their ability to describe such systems despite their complex structures. 
Moreover, the synthesis of observers becomes more interesting especially when we take into account defaults action on the dynamic and the output Equations (Johnson, 1975; Hostetter, 1973). This topic has been recently considered (Hou, 1992; Gaddouna, 1994; Darouach, 1996; Khadhraoui, 2014), and all related works estimate only the state vector. However, the proposed functional unknown input filter reconstructs both the functional state vector and the unknown input vector in the presence of faults. Then we can use these results in the failure detection and the control of systems in presence of disturbances.

Finally, the problem of the state observing of linear singular systems with bounded disturbances has been a great deal of investigation during the last decades (Khadhraoui, 2014; Darouach, 1996; Zasadzinski, 1998). In fact, filtering a state vector, lies on the reconstruction of a linear combination of the system states using only the input and the output measurements.

Two performance measures in filtering problem are extensively used, the $H_{2}$ and the $H_{\infty}$ norms (Khadhraoui, 2014, 2016; Souley, 2006). In this paper, we choose the $H_{\infty}$ filter due to its performance for minimizing the perturbation effect on the estimation error.

Motivated by these facts, we propose a functional $H_{\infty}$ filter to estimate both functional state vector and part of the unknown inputs vector based on Lyapunov-Krasovskii approach. And using the unbiasedness condition, we arrange the error expression so that the error dynamics ignores the disturbance derivative $(\dot{w}(t)$ ) (Souley, 2006). Then a Linear Matrix Inequalities (LMI) approach is developed to find the filter optimum gain.

The paper is organized as follows. Section 2 presents the difference between current work and recent published work. The third section gives assumptions used through this paper and presents the problem formulation that we propose to solve. Section 4 gives a time domain solution for the unknown input functional filter design problem with respect to the $H_{\infty}$ performance criterion. The next section summarizes the design algorithm presentation. Section 6 gives a numerical example to illustrate our approach and the last section concludes the paper.

\section{RELATED WORK}

In this paper, we propose to reconstruct; firstly, a functional state vector by reducing mathematical conditions and then reducing time calculate (Ezzine, 2010)). Secondly, in recent references (Khadhraoui, 2014, 2016) the filter of the unknown inputs systems which permit to avoid to have advanced perturbation term in the error dynamics; the authors propose an approach which resolves the $H_{\infty}$ filtering problem even when the term is present and they can estimate only the state vector. In this present paper we add of these previews results the estimation of the unknown inputs. This contribution is of major importance for faults detection.

\section{PROBLEM FORMULATION}

Let's consider the following continuous-time linear descriptor system described by:

$$
\begin{aligned}
& E \dot{x}(t)=A x(t)+B u(t)+F_{1} v(t)+G_{1} w(t) \\
& y(t)=C x(t)+D u(t)+F_{2} v(t)+G_{2} w(t)
\end{aligned}
$$


$z(t)=L x(t)$

where $x \in \mathbb{R}^{n}, u \in \mathbb{R}^{m}, y \in \mathbb{R}^{p}$ and $z \in \mathbb{R}^{r}$ are the state, the input, the output and the functional state vectors respectively. $v \in \mathbb{R}^{q}$ and $w \in \mathbb{R}^{k}$ are the unknown input and the bounded disturbance vectors. $E, A, B, F_{1}, F_{2}, G_{1}, G_{2}, L, D$ and $C$ are known matrices of appropriate dimensions.

In the sequel we suppose that:

Assumption 1: (Zasadzinski, 1998):

$\operatorname{rank}(E)=\bar{n}<n$

$\operatorname{rank}\left[\begin{array}{l}F_{1} \\ F_{2}\end{array}\right]=q$

$\operatorname{rank}\left[\begin{array}{l}G_{1} \\ G_{2}\end{array}\right]=k$

$\operatorname{rank}\left(F_{2}\right)=\bar{q}<q$

Then, there exist an orthogonal matrix $R \in \mathbb{R}^{p \times p}$ and a non singular matrix $R \in \mathbb{R}^{q \times q}$, such that:

$R^{T} F_{2} S=\left(\begin{array}{ll}I_{\bar{q}} & 0_{12} \\ 0_{21} & 0_{22}\end{array}\right)$

with $0_{12} \in \mathbb{R}^{\bar{q} \times(q-\bar{q})}, 0_{21} \in \mathbb{R}^{(p-\bar{q}) \times \bar{q}}$ and $0_{22} \in \mathbb{R}^{(p-\bar{q}) \times(q-\bar{q})}$.

Using Equation (2), system (1) is equivalent to:

$E \dot{x}(t)=\bar{A} x(t)+\bar{B} u(t)+F_{11} y_{1}(t)+F_{12} v_{2}(t)+\bar{G} w(t)$

$y_{1}(t)=C_{1} x(t)+D_{1} u(t)+v_{1}(t)+G_{11} w$

$y_{2}(t)=C_{2} x(t)+D_{2} u(t)+G_{22} w(t)$

$z(t)=L x(t)$

with: 
$\left[\begin{array}{l}y_{1}(t) \\ y_{2}(t)\end{array}\right]=R^{T} y(t)$ with $y_{1} \in \mathbb{R}^{\bar{q}}$ and $y_{2} \in \mathbb{R}^{p-\bar{q}}$

$\left[\begin{array}{l}v_{1}(t) \\ v_{2}(t)\end{array}\right]=S^{-1} v(t)$ with $v_{1} \in \mathbb{R}^{\bar{q}}$ and $v_{2} \in \mathbb{R}^{q-\bar{q}}$

$\left[\begin{array}{l}C_{1} \\ C_{2}\end{array}\right]=R^{T} C,\left[\begin{array}{l}D_{1} \\ D_{2}\end{array}\right]=R^{T} D,\left[\begin{array}{l}G_{11} \\ G_{22}\end{array}\right]=R^{T} G_{2}$

$\left[\begin{array}{ll}F_{11} & F_{12}\end{array}\right]=F_{1} S, \quad \bar{A}=A-F_{11} C_{1}$

$\bar{B}=B-F_{11} D_{1}, \quad \bar{G}=G_{1}-F_{11} G_{11}$

We note that Equations (3b) and (3c) are obtained by multiplying (1b) by $R^{T}$ and using (2).

\subsection{Objective}

The objective of this paper is to design in time domain an unknown input functional $H_{\infty}$ filter for linear singular system. The considered system is affected by a bounded disturbance acting on the dynamic and output Equations. The proposed filter reconstructs both the functional state $z(t)$ and the unknown input vector $v_{1}(t)$ derived from (3.b). So, we assume that:

$\bar{z}(t)=\left(\begin{array}{l}z(t) \\ v_{1}(t)\end{array}\right)$

The functional $H_{\infty}$ filter estimate $\bar{z}(t)$ described by Equation (6) use only the system known input $u(t)$ and output $y(t)$ as shown in Figure 1.

The system (3) can be written such:

$$
\begin{aligned}
& E \dot{x}(t)=\bar{A} x(t)+\bar{B} u(t)+F_{11} y_{1}(t)+F_{12} v_{2}(t)+\bar{G} w(t) \\
& y_{2}(t)=C_{2} x(t)+D_{2} u(t)+G_{22} w(t)
\end{aligned}
$$


Figure 1. The functional $H_{\infty}$ filter design

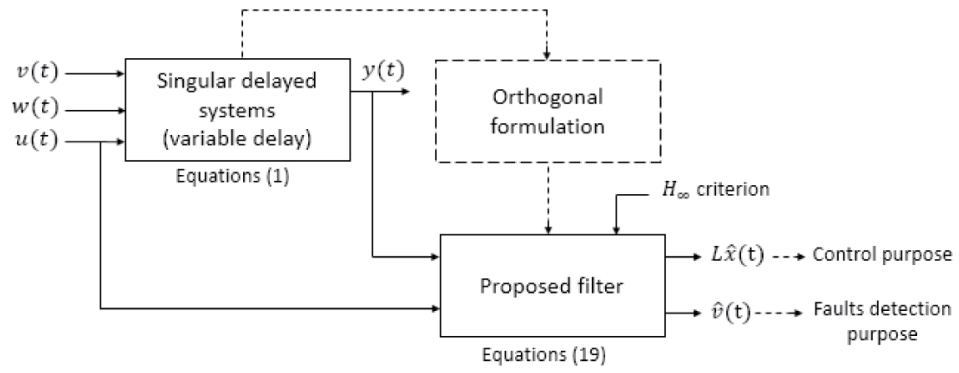

$\bar{z}(t)=\bar{L} x(t)+\bar{D}_{1} u(t)+\bar{G}_{11} w(t)+\bar{I}_{1}(t)$

with:

$$
\bar{L}=\left[\begin{array}{c}
L \\
-C_{1}
\end{array}\right], \bar{D}_{1}=\left[\begin{array}{c}
0_{r \times m} \\
-D_{1}
\end{array}\right], \bar{G}_{11}=\left[\begin{array}{c}
0_{r \times k} \\
-G_{11}
\end{array}\right], \bar{I}=\left[\begin{array}{c}
0_{r \times \bar{q}} \\
\bar{I}_{\bar{q}}
\end{array}\right]
$$

and:

$\bar{L} \in \mathbb{R}^{(r+\bar{q}) \times n}, \quad \operatorname{rank}(\bar{L})=r+\bar{q}<n$

In the sequel of the paper we suppose that: (Darouach, 1995):

$\operatorname{rank}\left(\begin{array}{c}E \\ C_{2} \\ \bar{L}\end{array}\right)=\operatorname{rank}\left(\begin{array}{c}E \\ C_{2}\end{array}\right)$

\section{TIME DOMAIN DESIGN}

From (10), there exists a non singular matrix $T$ :

$$
T=\left(\begin{array}{ll}
a & b \\
c & d
\end{array}\right)
$$

such as:

$$
a E+b C_{2}=\bar{L}
$$


$c E+d C_{2}=0_{(p-\bar{q}) \times n}$

with $a \in \mathbb{R}^{n \times n}, b \in \mathbb{R}^{n \times(p-\bar{q})}, c \in \mathbb{R}^{(p-\bar{q}) \times n}$ and $d \in \mathbb{R}^{(p-\bar{q}) \times(p-\bar{q})}$.

The designed functional filter is on the form:

$\dot{\eta}(t)=M \eta(t)+K u(t)+N_{1} y_{1}(t)+N_{2} y_{2}(t)$

$\hat{\bar{z}}(t)=\eta(t)+P u(t)+H_{1} y_{1}(t)+H_{2} y_{2}(t)$

where $\eta(t)$ is the state vector of the filter and $\hat{\bar{z}}(t)$ is the estimate of the functional state $\bar{z}(t)$. The matrices $M, K, N_{1}, N_{2}, P, H_{1}$ and $H_{2}$ will be determined using the LMI approach.

\subsection{Conditions of an Unknown Input Filter Synthesis}

Using (7c) and (14b) the estimation error $e(t)$ is given by:

$$
\begin{aligned}
& e(t)=\bar{z}(t)-\hat{\bar{z}}(t) \\
& =\left(\bar{L}-H_{2} C_{2}\right) x(t)+\left(\bar{D}_{1}-P-H_{2} D_{2}\right) u(t)+\left(\bar{G}_{11}-H_{2} G_{22}\right) w(t)+\left(\bar{I}-H_{1}\right) y_{1}(t)-\eta(t)
\end{aligned}
$$

We assume that:

$$
P=\bar{D}_{1}-H_{2} D_{2}
$$

$$
H_{1}=\bar{I}
$$

Then the Equation (15) becomes:

$$
e(t)=\left(\bar{L}-H_{2} C_{2}\right) x(t)+\left(\bar{G}_{11}-H_{2} G_{22}\right) w(t)-\eta(t)
$$

In order to avoid the disturbance time derivative $\dot{w}(t)$ in the error dynamics, we propose that:

$\epsilon(t)=\left(\bar{L}-H_{2} C_{2}\right) x(t)-\eta(t)$

$$
e(t)=\epsilon(t)+\left(\bar{G}_{11}-H_{2} G_{22}\right) w(t)
$$


Then, when replacing $\bar{L}$ by its expression (12) in (19a) and using Equation (13) we can write:

$$
\begin{aligned}
& \epsilon(t)=\left(a E+b C_{2}-H_{2} C_{2}\right) x(t)+\beta\left(c E+d C_{2}\right) x(t)-\eta(t) \\
& =(a+\beta c) E x(t)+\left(b+\beta d-H_{2}\right) C_{2} x(t)-\eta(t)
\end{aligned}
$$

where $\beta \in \mathbb{R}^{(r+\bar{q}) \times(p-\bar{q})}$.

We assume that:

$$
H_{2}=b+\beta d
$$

Then Equations (20) can be written in the following form:

$$
\epsilon(t)=(a+\beta c) E x(t)-\eta(t)=\varphi E x(t)-\eta(t)
$$

with:

$$
\varphi=a+\beta c
$$

Given the singular system (7) and the functional filter (14), we aim to design the matrices of the filter $M, K, N_{1}, N_{2}, P, H_{1}$ and $H_{2}$ such that:

$\lim _{t \rightarrow \infty} e(t)=0$, if $w=0$

The filtering error (19) is asymptotically stable and satisfy the $H_{\infty}$ performance.

The $H_{\infty}$ criterion is given by:

$0<H_{\epsilon w \infty}=\sup _{w \neq 0} \frac{\epsilon_{2}}{w_{2}}<\gamma$

with $H_{\epsilon w}(s)=\frac{\epsilon(s)}{w(s)}$ is a transfer matrix and $\gamma$ is a positive scalar.

For this, we propose the following theorem.

\subsubsection{Theorem 1}

The unbiasedness estimation error given by (19) relative to system (7) and functional $H_{\infty}$ filter (14) is verified such that:

$$
\dot{\epsilon}(t)=M \epsilon(t)+\left(\varphi \bar{G}-N_{2} G_{22}\right) w(t)
$$


$e(t)=\epsilon(t)+\left(\bar{G}_{11}-H_{2} G_{22}\right) w(t)$

if and only if the following Equations are satisfied:

$$
\begin{aligned}
& \varphi \bar{A}-N_{2} C_{2}-M \varphi E=0 \\
& \varphi \bar{B}-N_{2} D_{2}-K=0 \\
& \varphi F_{11}-N_{1}=0 \\
& \varphi F_{12}=0
\end{aligned}
$$

\subsubsection{Proof 1}

The derivative of $\epsilon(t)$ (22) is given as follows:

$\dot{\epsilon}(t)=\varphi E \dot{x}(t)-\dot{\eta}(t)$

Now, replacing (7a) and (14a) in (26), we obtain:

$$
\begin{aligned}
& \dot{\epsilon}^{\cdot}(t)=\varphi \bar{A} x(t)+\varphi \bar{B} u(t)+\varphi F_{11} y_{1}(t)+\varphi \bar{G} w(t)-M \eta(t)-K u(t)-N_{1} y_{1}(t) \\
& -N_{2} y_{2}(t)+\varphi F_{12} v_{-} 2(t)
\end{aligned}
$$

and:

$$
\begin{aligned}
& \epsilon^{\cdot}(t)=M \epsilon(t)+\left[\varphi \bar{A}-N_{2} C_{2}-M \varphi E\right] x(t)+\left[\varphi \bar{B}-N_{2} D_{2}-K\right] u(t) \\
& +\left[\varphi F_{11}-N_{1}\right] y_{1}(t)+\left[\varphi \bar{G}-N_{2} G_{22}\right] w(t)+\varphi F_{12} v_{2}(t)
\end{aligned}
$$

\subsection{Determination of Observer Matrices}

This method is based on the Lyapunov-Krasovskii stability theory (Krasovskii, 1963) and the LMI approach. So that, the stability conditions of the observer will be independent of the unknown input $v_{2}(t)$ and subsequently the estimated functional state $\hat{\bar{z}}(t)$ converges asymptotically to $\bar{z}(t)$.

By replacing (23) in conditions i) and iv) of theorem 1 and using (13), we have:

$$
a \bar{A}=M a E+J C_{2}-\beta c \bar{A}
$$

and: 
$a F_{12}=-\beta c F_{12}$

with:

$J=N_{2}-M \beta d$

Equations (29) and (30) can be written in matrix form:

$\Gamma=\Omega \Theta$

with:

$\Omega=\left[\begin{array}{lll}M & J & \beta\end{array}\right]$

$\Theta=\left(\begin{array}{cc}a E & 0 \\ C_{2} & 0 \\ -c \bar{A} & -c F_{12}\end{array}\right)$

$\Gamma=\left[a \bar{A} \quad a F_{12}\right]$

We note that the general Solution (33) exists if and only if:

$\operatorname{rank}\left[\begin{array}{l}\Theta \\ \Gamma\end{array}\right]=\operatorname{rank}(\Theta)$

Therefore, by respecting the Condition (36):

$\Omega=\Gamma \Theta^{+}-Z\left(I-\Theta \Theta^{+}\right)$

where $\Theta^{+}$is the generalized inverse of matrix $\Theta$ and $Z$ is an arbitrary matrix of appropriate size, that will be determined later using the LMI approach.

The unknown matrix $M$ is defined by:

$M=\Omega\left(\begin{array}{l}I \\ 0 \\ 0\end{array}\right)=M_{1}+Z M_{2}$ 
By replacing $\Omega$ by its expression given by (37) in (38), we obtain:

$M_{1}=\Gamma \Theta^{+}\left(\begin{array}{l}I \\ 0 \\ 0\end{array}\right), M_{2}\left(I-\Theta \Theta^{+}\right)\left(\begin{array}{l}I \\ 0 \\ 0\end{array}\right)$

Similarly, $M_{1}$ is obtained such:

$J=\Omega\left(\begin{array}{l}0 \\ I \\ 0\end{array}\right)=J_{1}+Z J_{2}$

By replacing $\Omega$ by its expression given by (37) in (40), we obtain:

$J_{1}=\Gamma \Theta^{+}\left(\begin{array}{l}0 \\ I \\ 0\end{array}\right), M_{2}\left(I-\Theta \Theta^{+}\right)\left(\begin{array}{l}0 \\ I \\ 0\end{array}\right)$

and $\beta$ is obtained such:

$\beta=\Omega\left(\begin{array}{l}0 \\ 0 \\ I\end{array}\right)=\beta_{1}+Z \beta_{2}$

By replacing $\Omega$ by its expression given by (37) in (42), we obtain:

$\beta_{1}=\Gamma \Theta^{+}\left(\begin{array}{l}0 \\ I \\ 0\end{array}\right), \beta_{2}\left(I-\Theta \Theta^{+}\right)\left(\begin{array}{l}0 \\ I \\ 0\end{array}\right)$

Using (31), (38) and (42) we can write $N_{2} G_{22}$ such that:

$$
\begin{aligned}
& N_{2} G_{22}=(J+M \beta d) G_{22} \\
& =J G_{22}+\left(M_{1}+Z M_{2}\right)\left(\beta_{1}+Z \beta_{2}\right) d G_{22} \\
& =J G_{22}+M_{1}\left(\beta_{1}+Z \beta_{2}\right) d G_{22}+\left(Z M_{2} \beta_{1}+Z M_{2} Z \beta_{2}\right) d G_{22}
\end{aligned}
$$


Since the matrix $N_{2} G_{22}$ have a bi-linearity $\left(Z M_{2} Z\right)$. So we assume that the gain matrix $Z$ satisfies the following relation:

$$
Z \beta_{2}=0
$$

So there always exists a matrix $Z_{1}$ such that:

$Z=Z_{1}\left(I-\beta_{2} \beta_{2}^{+}\right)=Z_{1} \Delta$

Then $M, J$ and $\beta$ are defined as:

$$
\begin{aligned}
& M=M_{1}+Z_{1} \Delta M_{2} \\
& J=J_{1}+Z_{1} \Delta J_{2} \\
& \beta=\beta_{1}+Z_{1} \Delta \beta_{2}
\end{aligned}
$$

At this stage, we propose to replace in (25) $H_{2}, \varphi$ and $N_{2} G_{22}$ by their expressions given by (21), (23) and (44) respectively, we can write:

$$
\epsilon^{\cdot}(t)=A_{0} \epsilon(t)+B_{0} w(t)
$$

$$
e(t)=C_{0} \epsilon(t)+D_{0} w(t)
$$

where:

$$
\begin{aligned}
& A_{0}=M \\
& B_{0}=\left(a+\beta_{1} c\right) \bar{G}-\left(J_{1}+M_{1} \beta_{1} d\right) G_{22}+Z_{1} \Delta\left[\beta_{2} c \bar{G}-\left(J_{2}+M_{2} \beta_{1} d\right) G_{22}\right] \\
& C_{0}=I
\end{aligned}
$$


$D_{0}=\bar{G}_{11}-\left(b+\beta_{1} d\right) G_{22}$

Now we can determine all matrix of filter (14) if and only if the matrix $Z_{1}$ is determined, for this we use the following theorem.

\subsubsection{Theorem 2}

The system (14) is a functional filter for the system (1) if there exist matrices $P=P^{T}>0$ and $Y$ which satisfy the following matrix inequality:

$$
\left(\begin{array}{ccc}
A_{0}^{T} P+P A_{0} & P B_{0} & C_{0}^{T} \\
* & -\gamma^{2} I & D_{0}^{T} \\
* & * & -I
\end{array}\right)<0
$$

The gain $Z_{1}$ is given by:

$$
Z_{1}=P^{-1} Y
$$

\subsubsection{Proof 2}

Let $V(\epsilon, t)$ be the Lyapunov-Krasovskii functional of the form:

$V(\epsilon, t)=\epsilon^{T}(t) P \epsilon(t)$

where $P=P^{T}>0$.

In order to establish sufficient conditions for existence of (14) according to (24), we should verify the following inequality:

$$
H(\epsilon, t)=\dot{V}(\epsilon, t)+e^{T}(t) e(t)-\gamma^{2} w^{T}(t) w(t)<0
$$

Using (51b), Equation (59) can be written as:

$$
\left(\begin{array}{ll}
\epsilon^{T} & w^{T}
\end{array}\right)\left(\begin{array}{cc}
A_{0}^{T} P+P A_{0}+C_{0}^{T} C_{0} & P B_{0}+C_{0}^{T} D_{0} \\
* & D_{0}^{T} D_{0}-\gamma^{2} I
\end{array}\right)\left(\begin{array}{l}
\epsilon \\
w
\end{array}\right)<0
$$

According to the Schur Lemme (Khadhraoui, 2016) we deduct Equation (55).

\section{ALGORITHM FOR FUNCTIONAL FILTER DESIGN}

Step 1: Check that conditions 1), 2), 3) and 4) of assumption 1 are satisfied.

Step 2: Compute matrices $R$ and $\mathrm{S}$ using (2). 
Step 3: Check that condition (10) is verified.

Step 4: Compute matrix $T$ using (12) and (13).

Step 5: Compute $\Theta$ and $\Gamma$ using (34) and (35).

Step 6: Deduce the values of matrices $M_{1}, M_{2}, J_{1}, J_{2}, \beta_{1}$ and $\beta_{2}$ from (39), (41) and (43).

Step 7: Verify that the condition (36) is satisfied. Then resolution of the proposed LMI (55), gives the gain matrix $Z_{1}$.

Step 8: Compute, $J$, and $\beta_{1}$ using (47), (48) and (49).

Step 9: Get matrices $P, H_{1}, H_{2}$ and $N_{2}$ using (16), (17), (21) and (31).

Step 10: Get matrices $K$ and $N_{1}$ using ii) and iii) from theorem 1.

\section{NUMERICAL EXAMPLES}

Let's consider system (1) where:

$$
\begin{aligned}
& E=\left(\begin{array}{ccc}
1 & 0 & 0 \\
-2 & 0 & 0 \\
0 & 0 & 1
\end{array}\right), A=\left(\begin{array}{ccc}
1 & -1 & -1 \\
2 & -1 & 1 \\
2 & 0 & -1
\end{array}\right), C=\left(\begin{array}{lll}
2 & 1 & 0 \\
0 & 1 & 0 \\
1 & 1 & 1
\end{array}\right), B=\left(\begin{array}{l}
0 \\
1 \\
0
\end{array}\right) \\
& F_{1}=\left(\begin{array}{ll}
1 & 0 \\
1 & 2 \\
0 & 1
\end{array}\right), F_{2}=\left(\begin{array}{ll}
0 & 0 \\
1 & 2 \\
0 & 0
\end{array}\right), G_{1}=\left(\begin{array}{c}
1 \\
-1 \\
1
\end{array}\right), G_{2}=\left(\begin{array}{l}
5 \\
1 \\
0
\end{array}\right), L=\left(\begin{array}{lll}
1 & -1 & 1
\end{array}\right)
\end{aligned}
$$

The $H_{\infty}$ criterion is bounded by $=1,5$.

The input signal $u(t)$, the bounded disturbance $w(t)$ and the unknown inputs $u(t)$ are given by Figures 2-4 respectively.

Following the procedure proposed below, we obtain:

$$
S=\left(\begin{array}{ll}
-0.2 & -0.89 \\
-0.4 & -0.44
\end{array}\right), R=\left(\begin{array}{ccc}
0 & -1 & 0 \\
-1 & 0 & 0 \\
0 & 0 & 1
\end{array}\right)
$$

Figure 2. Input signal $u(t)$

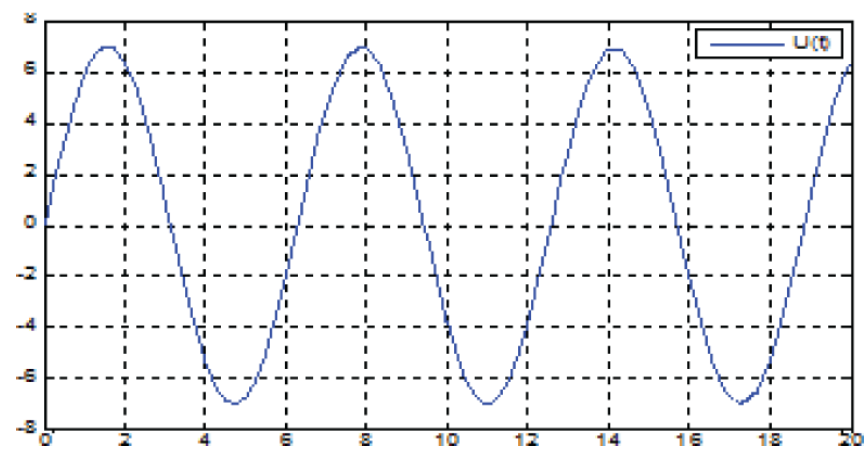


Figure 3. Bounded disturbance $w(t)$

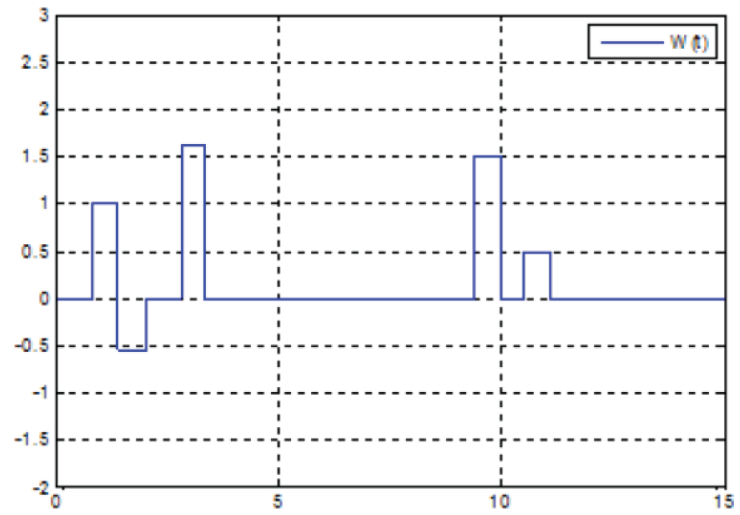

Figure 4. Unknown inputs $v(t)$
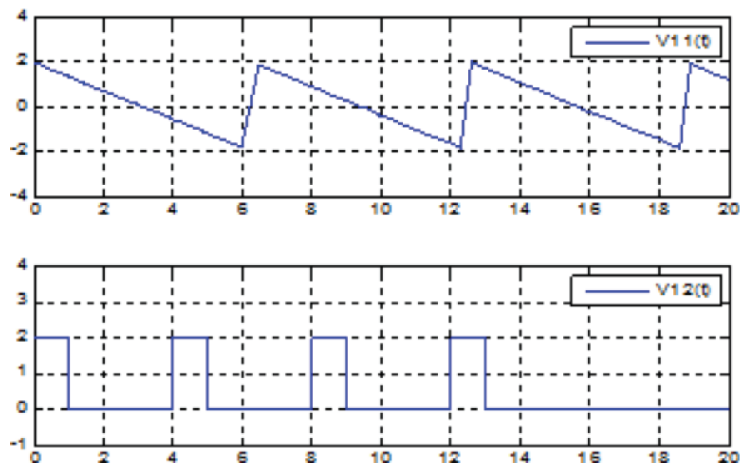

By multiplying the unknown inputs $v(t)$ by $S^{-1}$ we have (Figure 5):

The LMI approach application (55) yields:

$$
\begin{aligned}
& M=\left(\begin{array}{cc}
-5.54 & -9.25 \\
2.74 & 3.79
\end{array}\right), K=\left(\begin{array}{c}
-2.44 \\
1.59
\end{array}\right), N_{1}=\left(\begin{array}{c}
0.43 \\
-0.45
\end{array}\right), N_{2}=\left(\begin{array}{cc}
0.94 & -0.06 \\
-0.56 & 0.43
\end{array}\right) \\
& H_{1}=\left(\begin{array}{l}
0 \\
1
\end{array}\right), H_{2}=\left(\begin{array}{cc}
0.75 & -0.24 \\
-0.56 & 0.43
\end{array}\right), P=\left(\begin{array}{c}
1.75 \\
-0.56
\end{array}\right)
\end{aligned}
$$

Figure 6 represents the evolution of the real and the estimated functional state vector $z(t)$ and Figure 7 represents the real and the estimated unknown inputs $v_{1}(t)$.

We can remark as it shown in Figure 6 and Figure 7 the time domain behavior of the functional $H_{\infty}$ filter. The proposed filter can estimate the functional state and the unknown inputs vectors independently of unknown inputs with respect to the disturbance attenuation criterion; the effect of the disturbance on the estimation error is evaluated by $H_{e w \infty}=1,3<\gamma$. 
Figure 5. Part of unknown inputs $v_{1}(t)$

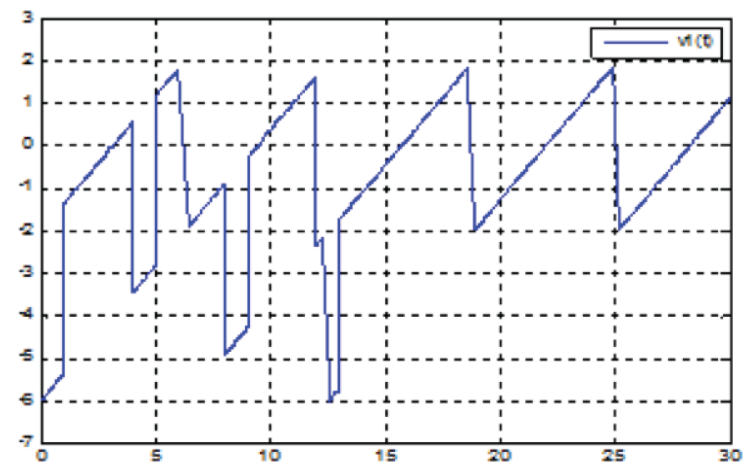

Figure 6. Functional state $z(t)$

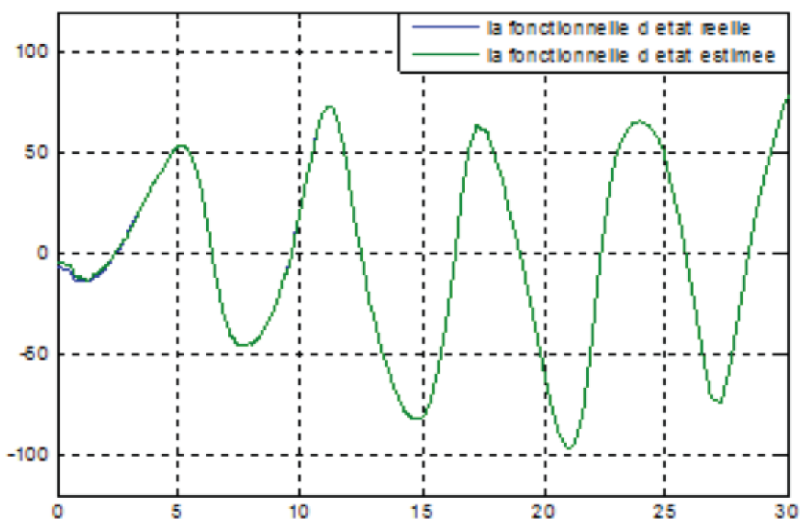

Figure 7. Unknown inputs $v_{1}(t)$

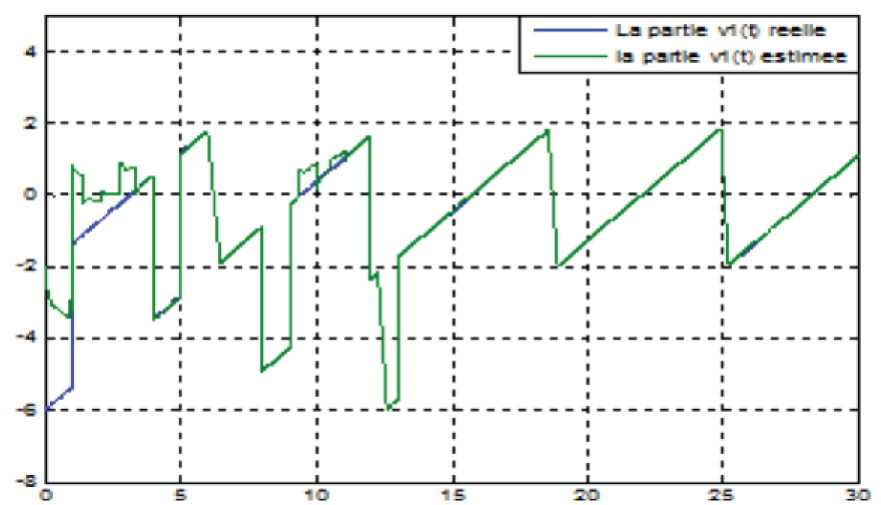


Then the estimation error of the functional state $z(t)$ and the unknown input $v_{1}(t)$ are given by Figure 8 and Figure 9 respectively.

These figures show the disturbance effect on the estimation error of functional state and unknown inputs vectors respectively during the Transient phase, permanent phase and the Transient duration when disturbance is applied ( $H_{e w \infty}=1,3<\gamma$ ). So, we conclude the convergence of the error dynamic witch confirm the effectiveness of the proposed approach.

Figure 8. Estimation error of functional state

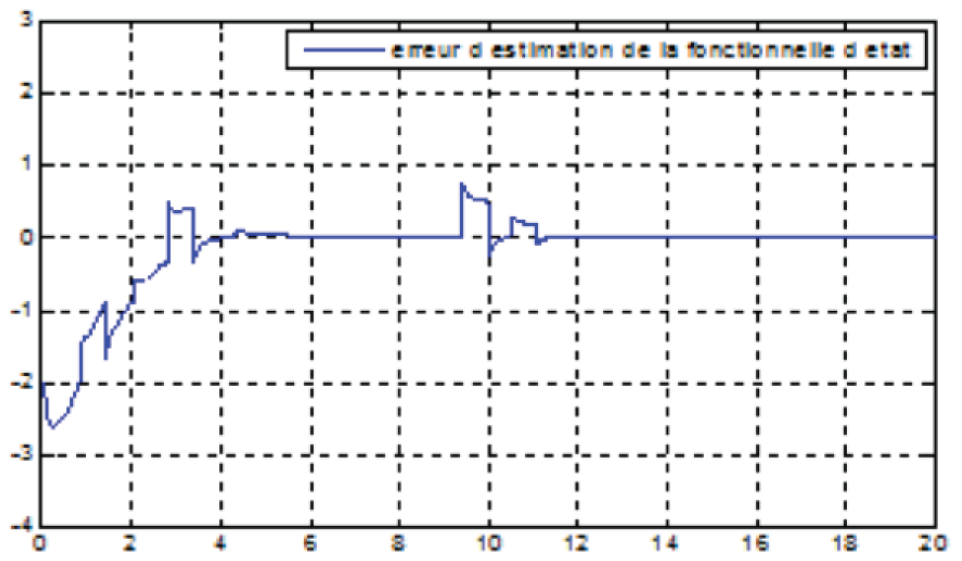

Figure 9. Estimation error of unknown input

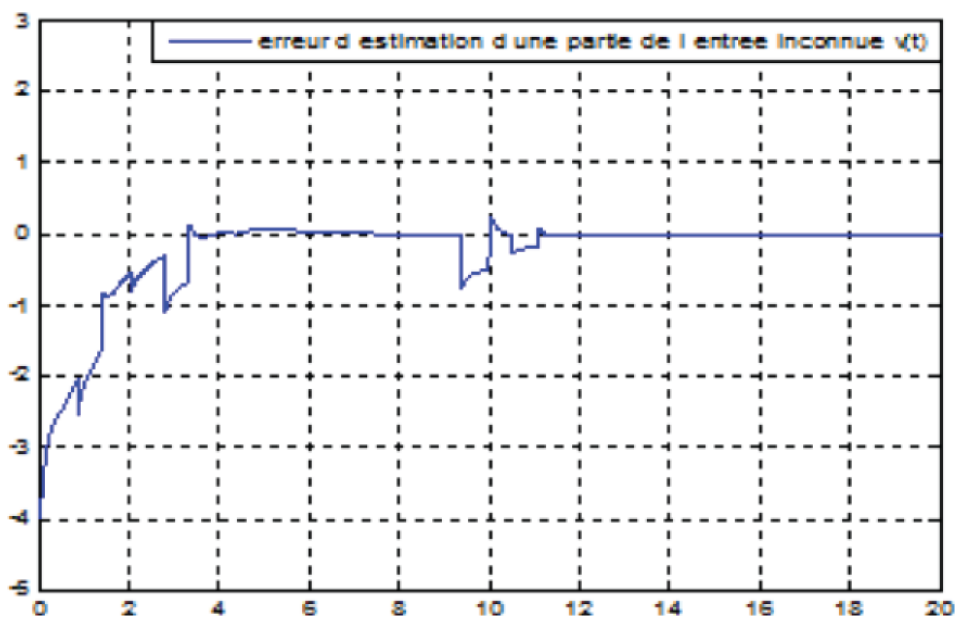

\section{CONCLUSION}

In this paper, a new method for functional $H_{\infty}$ filter of linear singular systems with an additional unknown inputs and bounded disturbances has been established in time domain. The output of the proposed filter combines the state and the unknown input vectors. 
An LMI solution is proposed to find the optimum gain implemented in the observer design. This solution is based on the unbiasedness of estimation error, the Lyapunov function approach (Krasovskii, 1963) and the disturbance minimizing effect on the estimation error.

Thanks to this filter we can estimate a part of the unknown inputs in order to detect some defaults. 


\section{REFERENCES}

Balochian, S., \& Nahid, R. (2018). Fractional-Order Optimal Control of Fractional-Order Linear Vibration Systems with Time Delay. International Journal of System Dynamics Applications, 7(3), 72-93. doi:10.4018/ IJSDA.2018070104

Bouzaida, S., \& Sakly, A. (2018). Adaptive Neuro-Fuzzy Sliding Mode Controller. International Journal of System Dynamics Applications, 7(2), 34-54. doi:10.4018/IJSDA.2018040103

Boyd, S. P., El Ghaoui, L., Féron, E., \& Balakrishnan, V. (1994). Linear Matrix Inequality in Systems and Control Theory. SIAM. doi: $10.1137 / 1.9781611970777$

Dai, L. (1989). Singular Control Systems. In Lecture Notes in Control and Information Sciences (vol. 118). Springer-Verlag.

Darouach, M., \& Boutayeb, M. (1995). Design of observers for descriptor systems. IEEE Transactions on Automatic Control, 40(7), 1323-1327. doi:10.1109/9.400467

Darouach, M., Zasadzinski, M., \& Hayar, M. (1996). Reduced-Order Observer Design for Descriptor Systems with Unknown Inputs. IEEE Transactions on Automatic Control, 41(7), 1068-1072. doi:10.1109/9.508918

Darouach, M. (2005). Linear Functional Observers for systems with delays in state variables: The discrete time case. IEEE Transactions on Automatic Control, 50(2), 228-233. doi:10.1109/TAC.2004.841932

Ding, X., Guo, L., \& Frank, M. (1994). Parameterization of linear observers and its application to observer design. IEEE Transactions on Automatic Control, 39(8), 1648-1652. doi:10.1109/9.310042

Ezzine, M., Darouach, M., Souley, H., \& Messaoud, H. (2010). Synthese temporelle et frequentielle de filtres d'ordre plein pour les systemes singuliers. Conference Internationale Francophone d'Automatique.

Ezzine, M., Darouach, M., Souley, H., \& Messaoud, H. (2011). Unknown Inputs Functional Observers Designs For Descriptor Systems with Constant Time Delay. IFAC, 44(1), 1162-1167.

Ezzine, M., Darouach, M., Souley, H., \& Messaoud, H. (2012). A controller design based on a functional $\mathrm{H}_{\infty}$. Automatica, 48(3), 542-549. doi:10.1016/j.automatica.2011.08.060

Gaddouna, B., Maquin, D., \& Rago, J. (1994). Fault detection observer design for linear systems with unknown inputs. IFAC, 27(5), 59-64.

Ghabi, J., Rhif, A., \& Vaidyanathan, S. (2018). Discrete Time Sliding Mode Control Scheme for Nonlinear Systems With Bounded Uncertainties. International Journal of System Dynamics Applications, 7(2), 15-33. doi:10.4018/IJSDA.2018040102

Hou, M., \& Müller, P. C. (1992). Design of observers for linear systems with unknown inputs. IEEE Transactions on Automatic Control, 37(6), 871-875. doi:10.1109/9.256351

Hostetter, G., \& Meditch, J. (1973). Observing systems with unmeasurable inputs. IEEE Transactions on Automatic Control, 18(3), 307-308. doi:10.1109/TAC.1973.1100296

Johnson, C. (1975). On observers for systems with unknown and inaccessible inputs. International Journal of Control, 21(5), 825-831. doi:10.1080/00207177508922036

Khadhraoui, M., Ezzine, M., Messaoud, H., \& Darouch, M. (2014). Design of full order filter for delayed singular systems with unknown input and bounded disturbance. International Conference on Control, Decision and Information Technologies, 429-434.

Khadhraoui, M., Ezzine, M., \& Messaoud, H. (2014). Design of Full Order Observers with Unknown Inputs for Delayed Singular Systems with Constant Time Delay. International Conference on Control, Decision and Information Technologies, 423-428.

Khadhraoui, M., Ezzine, M., \& Messaoud, H. (2016). A controller design based on a functional $\mathrm{H}_{\infty}$ filter for delayed singular systems: The time and frequency domain cases. WSEAS Transactions on Systems, 48(3), $542-549$. 
Koenig, D., \& Marx, B. (2004). Design of Observers for descriptor systems with delayed state and unknown inputs. American Control Conference, 5(5), 4806-4810. doi:10.23919/ACC.2004.1384073

Kurek, J. (1983). The state reconstruction for linear systems with unknown inputs. IEEE Transactions on Automatic Control, 28(12), 1120-1122. doi:10.1109/TAC.1983.1103189

Krasovskii, N. (1963). Stability of Motion. Stanford University Press.

Masubuchi, I., Kamitane, Y., Ohara, A., \& Suda, N. (1997). Control for descriptor systems: A matrix inequalities approach. Automatica, 33(4), 669-673. doi:10.1016/S0005-1098(96)00193-8

Meziane, S., Atarsia, L., \& Toufouti, R. (2018). A Global Stability of Linearizing Control of Induction Motor for PV Water Pumping Application. International Journal of System Dynamics Applications, 7(3), 31-56. doi:10.4018/IJSDA.2018070102

Verghese, G., Levy, B., \& Kailath, T. (1981). A generalized state-space for singular systems. IEEE Transactions on Automatic Control, 26(4), 811-831. doi:10.1109/TAC.1981.1102763

Yang, F., \& Wilde, R. W. (1988). Observers for linear systems with unknown inputs. IEEE Transactions on Automatic Control, 33(7), 677-681. doi:10.1109/9.1278

Seyed, H. N., \& Saeed, B. (2018). The Stability of a Class of Fractional Order Switching System with Time-Delay Actuator. International Journal of System Dynamics Applications, 7(1), 85-96. doi:10.4018/IJSDA.2018010105

Souley, H. Darouach, M.\& Zasadzinski, M. (2006). Approche LMI pour la synthèse des filtres $\mathrm{H}_{\infty}$ non Biaisés. Academic Press.

Zasadzinski, M., Rafaralahy, H., Mechmeche, C., \& Darouach, M. (1998). On Disturbance Decoupled Observers for a Class of Bilinear System. Journal of Dynamic Systems, Measurement, and Control, 120(3).

Zerrougui, M. (2011). Observation et Commande des Systemes Singuliers non Lineaires ( $\mathrm{PhD}$ thesis). Université de Henry Poincaré-Nancy 1, France. 